\title{
Water structure near single and multi-layer nanoscopic hydrophobic plates of varying separation and interaction potentials
}

\author{
MALAY RANA and AMALENDU CHANDRA* \\ Department of Chemistry, Indian Institute of Technology, Kanpur 208 016, India
}

\begin{abstract}
We have performed a series of molecular dynamics simulations of water containing two nanoscopic hydrophobic plates to investigate the modifications of the density and hydrogen bond distributions of water in the vicinity of the surfaces. Our primary goal is to look at the effects of plate thickness, solutesolvent interaction and also interplate separation on the solvent structure in the confined region between two graphite-like plates and also near the outer surfaces of the plates. The thickness of the plates is varied by considering single and triple-layer graphite plates and the interaction potential is varied by tuning the attractive strength of the 12-6 pair interaction potential between a carbon atom of the graphite plates and a water molecule. The calculations are done for four different values of the tuning parameter ranging from fully LennardJones to pure repulsive pair interactions. It is found that both the solvation characteristics and hydrogen bond distributions can depend rather strongly on the strength of the attractive part of the solute-water interaction potential. The thickness of the plates, however, is found to have only minor effects on the density profiles and hydrogen bond network. This indicates that the long range electrostatic interactions between water molecules on the two opposite sides of the same plate do not make any significant contribution to the overall solvation structure of these hydrophobic plates. The solvation characteristics are primarily determined by the balance between the loss of energy due to hydrogen bond network disruption, cavity repulsion potential and offset of the same by attractive component of the solute-water interactions. Our studies with different system sizes show that the essential features of solvation properties, e.g. wetting and dewetting characteristics for different interplate separations and interaction potentials, are also present in relatively smaller systems consisting of a few hundred atoms.
\end{abstract}

Keywords. Water structure; hydrogen bonds; hydrophobic surfaces; dispersion and repulsive interactions; nanomaterials.

\section{Introduction}

A molecular-level understanding of the solvation of nanoscopic hydrophobic surfaces is extremely important in many diverse areas of chemistry, physics, biology and materials science and, therefore, it is not surprising that investigations of such hydrophobic surfaces in contact with an aqueous medium have received much attention in the recent past (Huang and Chandler 2000, 2002; Hummer et al 2000, 2001; Wallqvist et al 2001; Gordillo and Marti 2002; Beckstein and Sansom 2003; Huang et al 2003; Chowdhury and Pettitt 2005a, b). Unlike smaller hydrophobic solutes (Smith and Haymet 1993; Lum et al 1999), bigger nano-sized solutes cannot be accommodated in cavities of atomic dimensions that are spontaneously created in liquid water and thus solvation of nanoscopic hydrophobic solutes require significant disruption of water hydrogen bonding network in its vicinity. In spite of many recent theoretical and computational studies on solvation of large hydrophobic solutes in liquid water

\footnotetext{
*Author for correspondence (amalen@iitk.ac.in)
}

(Huang and Chandler 2000, 2002; Hummer et al 2000, 2001; Wallqvist et al 2001; Gordillo and Marti 2002; Beckstein and Sansom 2003; Huang et al 2003; Chowdhury and Pettitt 2005a, b), our understanding of the molecular details of hydrophobic solvation of nanoscopic surfaces is far from complete.

Because of the inherent complexity of hydrophobic surfaces and the associated solvation processes, it is useful to consider simplified models, examples include structureless planar hard walls, soft walls with both repulsive and attractive components for surface-water interactions, large solutes of spherical or ellipsoidal shapes and atomistic surfaces consisting of neutral atoms of varying interaction potentials. For purely repulsive model solutes of nanoscopic dimensions, it has been found that water molecules could be expelled from the intervening region between two such solutes when the separation between the solutes is less than a critical distance (Wallqvist et al 2001; Huang et al 2003). The solute-water repulsion, disruption of hydrogen bond network and associated loss of energy coupled with cavity repulsion potential due to the attractive hydrogen bonded interactions of surface water with those on the bulk side give rise to a dewetting 
of the fully repulsive hydrophobic solutes of nanoscopic dimensions. However, this dewetting effect was found to decrease upon inclusion of an attractive interaction between the solutes and the water molecules (Wallqvist et al 2001; Huang et al 2003). Recent atomistic simulations have provided a more detailed picture of the solvation of nano-sized hydrophobic plates in water (Hummer et al 2001; Beckstein and Sansom 2003; Chowdhury and Pettitt 2005a, b). In some of these studies (Chowdhury and Pettitt 2005a, b), atomistic model graphite-like plates were immersed in model liquid water and wetting/ dewetting behaviour of the plates was studied for different interaction potentials starting from fully Lennard-Jones with some reasonable parameter values for the attractive interaction to purely repulsive interactions. Although solvation of a large hydrophobic plate is not expected to be a favourable process in general, a nice balance between several factors lead to a wetting of the graphite-like plates in presence of attractive solute-water interactions and dewetting of the same when the solute-water interaction is modelled by purely repulsive potential. Of course, apart from the repulsive or attractive nature of the solutewater interactions, other parameters such as the separation between the plates was also found to be important in determining the wetting and dewetting characteristics of hydrophobic plates (Chowdhury and Pettitt 2005a).

The existing studies on the effects of interaction potentials on solvation of graphite plates have considered either one single-layer graphite plate or two such single-layer plates kept at an interplate separation of few Angstroms. Although a single-layer plate is sufficient for the disruption of hydrogen bond network and for the creation of cavity repulsion potential, water molecules are also known to have long range interactions which can be significant even beyond the typical length scale of a single-layer plate which is about $3 \AA$. Because of the presence of long-range electrostatic interactions, water molecules on one side of the plate can interact with those on the other side of the same plate and it is not clear how much these long-range interactions between water molecules on two opposite sides of a plate contribute or influence its solvation characteristics, especially in the context of wetting and dewetting transitions. Compared to the case of a single-layer plate, water molecules on one side of a multilayer plate would be further away from those on the other side and so interactions would also be much weaker with those on the other side. Thus, it would be interesting to investigate the solvation of graphite plates with different thicknesses, starting from single-layer to multi-layer, and see how the solvation characteristics change with changes of thickness of the graphite plates. Such a study is presented here. In addition, we also look at the effects of system size and solute-solvent attractive interaction potential on the wetting and dewetting characteristics of both single and multi-layer graphite plates. We note that although the density profiles of water near single-layer graphite plates of varying attractive strength were also calculated in some of the recent studies (Chowdhury and Pettitt 2005a, b), we preferred to calculate these quantities again in the present work so that the results for these single-layer plates can be directly compared with those for the multilayer plates under identical simulation conditions. Since the simulation conditions and also the tuning of the solutewater attractive potential in these earlier studies were different from those considered here, it would be meaningful to compare the present results for single-layer graphite plates with those of earlier studies to judge the robustness of the main observations with respect to the variations of simulation conditions and interaction potentials.

The present calculations reveal that the structure of water near a graphite plate depends rather weakly on its thickness. Calculations with single and triple-layer plates produce very similar density profiles and hydrogen bonded structure of water molecules between the two plates and also near the outer surfaces of the plates. On the other hand, both the solvation characteristics and hydrogen bond distributions are found to depend rather strongly on the strength of the attractive part of the solute-water interaction potential. This indicates that the solvation characteristics are primarily determined by the net effects of energy loss due to hydrogen bond network disruption, creation of cavity repulsion potential and offset of the same by attractive solute-water interactions. The long range electrostatic interactions between water molecules on the two opposite sides of the same plate do not seem to make any significant influence on the overall solvation structure of these hydrophobic plates. Our calculations with different system sizes show that the essential features of solvation properties, e.g. wetting and dewetting characteristics for different interplate separations and interaction potentials, are also present in relatively smaller systems consisting of a few hundred atoms. This is an important result in view of the fact that all of the present and previous simulations of water near graphite surfaces are based on empirical pair potentials for graphite-water interactions. However, the true interaction potential between a graphite plate and a water molecule is not known and so the smaller systems of the present study can be employed for more accurate calculations, ab initio molecular dynamics for example, to understand the nature of hydrophobic interactions in a better way.

We have organized rest of the paper as follows. In $\S 2$, we describe the models of water and different hydrophobic plates that are employed here and also other details of simulations. In $\$ 3$, we have presented the results of the density and hydrogen bond distributions of water in the confined regions and also in the vicinity of the outer surfaces of the two hydrophobic plates for different interaction potentials. Results are presented for both single and multi-layer plates and also for two different system sizes. Our conclusions are briefly summarized in $\S 4$. 


\section{Model and simulation details}

Our simulation systems consist of two hydrophobic graphitelike plates immersed in liquid water. The interplate separation and also the thickness and attractive strength of the two plates are varied over wide ranges. In all of our present simulations, the water molecules are represented by the well known SPC/E model (Berendsen et al 1987), where the interaction between atomic sites of two water molecules is given by

$$
u_{\mathrm{ww}}\left(r_{i}, r_{j}\right)=\left(\frac{A_{i j}}{r_{i j}}\right)^{12}-\left(\frac{B_{i j}}{r_{i j}}\right)^{6}+\frac{q_{i} q_{j}}{r_{i j}},
$$

where, $q_{i}$ is the charge of the $i$ th atom of a water molecule and $A_{i j}$ and $B_{i j}$ are the coefficients of the short range 12-6 interactions which are non-zero only for oxygen-oxygen interactions and zero for interactions with hydrogen atoms. For oxygen-oxygen interactions, the values of $A_{i j}$ and $B_{i j}$ are $3.428\left(\mathrm{~kJ} \mathrm{~mol}^{-1}\right)^{1 / 12} \AA$ and $3.7122\left(\mathrm{~kJ} \mathrm{~mol}^{-1}\right)^{1 / 6} \AA$, respectively. We note that these values of $A_{i j}$ and $B_{i j}$ correspond to a Lennard-Jones well-depth of $0.6502 \mathrm{~J} \mathrm{~mol}^{-1}$ and diameter of $3.169 \AA$. The partial charges on the oxygen and hydrogen atoms are $-0.8476 e$ and $+0.4238 e$, respectively, where $e$ is the magnitude of the electronic charge.

Each of the hydrophobic plates is modelled as a single or multi-layer graphite sheet where, in each layer, carbon atoms are placed in the hexagonal lattice positions with a carbon-carbon distance of $1.4 \AA$. An interlayer spacing of $3.4 \AA$ is used for multi-layer plates. The carbon atoms are kept fixed at their lattice positions so that only the carbonwater interactions and forces need to be considered in the present simulations. Since the carbon atoms are neutral without any partial charges, it is assumed that only the short-range interaction with the oxygen atoms needs to be considered for carbon-water interactions and it is modelled by the following potential

$$
u_{\mathrm{CO}}\left(r_{i}, r_{j}\right)=\left(\frac{A_{\mathrm{CO}}}{r_{i j}}\right)^{12}-\lambda\left(\frac{B_{\mathrm{CO}}}{r_{i j}}\right)^{6},
$$

where $A_{\mathrm{CO}}=3.470 \quad\left(\mathrm{~kJ} \mathrm{~mol}^{-1}\right)^{1 / 12} \AA, \quad B_{\mathrm{CO}}=3.666 \quad(\mathrm{~kJ}$ $\left.\mathrm{mol}^{-1}\right)^{1 / 6} \AA$, and $\lambda$ is a parameter that determines the weight of the attractive dispersion part of the carbonoxygen interaction potential. We note that, for $\lambda=1$, the above potential is equivalent to a Lennard-Jones interaction between graphite carbon and water oxygen atoms with a well-depth parameter of $0.3598 \mathrm{~kJ} \mathrm{~mol}^{-1}$ and a diameter of $3.4 \AA$ for carbon atoms. These Lennard-Jones parameter values correspond to AMBER force fields (Cornell et al 1995) for a $s p^{2}$ carbon atom and have been used in many of the earlier studies of water near graphite surfaces and carbon nanotubes. In the present work, we have varied the values of $\lambda$ from 0 to 1 to tune the potential from a purely repulsive to a fully Lennard-Jones inter- action and thus to study the role of the attractive dispersion component in the solvation of hydrophobic plates.

First, we have simulated a system of two single-layer graphite plates, each consisting of 28 carbon atoms and placed symmetrically around the centre of the simulation box with an interplate separation $(d)$ of $6.8 \AA$ along the $z$ direction, immersed in a system of 500 water molecules. The size of the box is determined by the density of water at room temperature and, upon insertion of the graphite plates, overlapping water molecules are removed from the simulation systems and the resultant system containing the graphite plates are reequilibrated. Calculations are done for four different values of $\lambda$ that characterize the strength of the attractive part of the carbon-water interactions.

Simulations are also done for a single carbon atom immersed in 499 water for the same values of the $\lambda$ parameter. Subsequently, we considered bigger systems so that larger interplate separations could be achieved. Specifically, we have considered three systems with two single layer graphite plates dipped in water of 2048 molecules with interplate separations of $13,9.8$ and $6.8 \AA$ and with 60 carbon atoms in each plate and another three systems with two triple-layer graphite plates dipped in 2048 water molecules with interplate separations (perpendicular distance between the closest layers of the two plates) of 13, 9.8 and $6.8 \AA$ and with 180 carbon atoms in each plate. Again, for all the systems, overlapping water molecules are removed upon insertion of the graphite plates and the resultant systems are reequilibrated for sufficiently long time. Also, these later calculations of larger systems are done for two different values of the tuning parameter: $\lambda=1$ and $\lambda=0$. The choice of the interplate separations of $6 \cdot 8,9 \cdot 8$ and $13 \AA$ was guided by an earlier study which reported local minima of potential of mean force between two solvated single-layer graphite-like plates at these interparticle separations corresponding to one, two and three intervening water layers, respectively between the two plates. Finally, we have also simulated a smaller system containing two single-layer graphite plates of 28 atoms each $(d=6.8 \AA)$ and 148 water molecules (after removing the overlapping molecules) to see if the essential aspects of the solvation characteristics that are observed for larger systems are also exhibited by the smaller systems of a few hundred atoms. These smaller system simulations are also done for two different values of $\lambda$ and thus, altogether, we have simulated 22 different systems in the present study.

In all simulations, we have employed a minimum image convention for the short-range interaction potential and the water-water long-range electrostatic interactions are treated using the Ewald method (Allen and Tildesley 1987). We employed the quaternion formulation of the equations of rotational motion and, for the integration over time, we adapted the leap-frog algorithm with a time-step of $10^{-15} \mathrm{~s}(1 \mathrm{fs})$. For each system, we first ran 

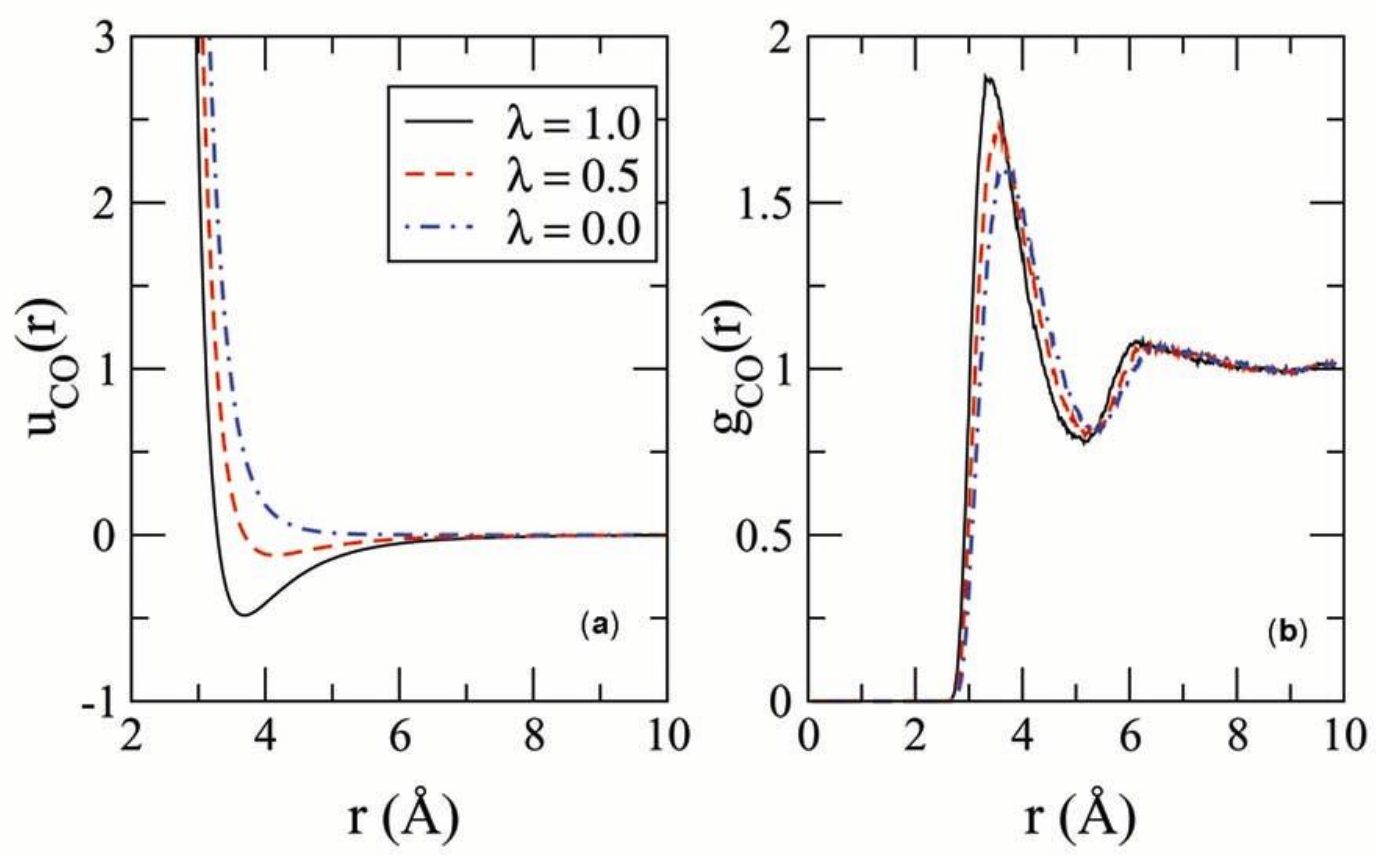

Figure 1. (a) Carbon-water interaction potential as given by (2) for three different values of the tuning parameter, $\lambda$ and (b) carbon-oxygen radial distribution functions obtained from simulations of a single carbon atom in water for different values of $\lambda$. The different curves in (b) are as in (a).
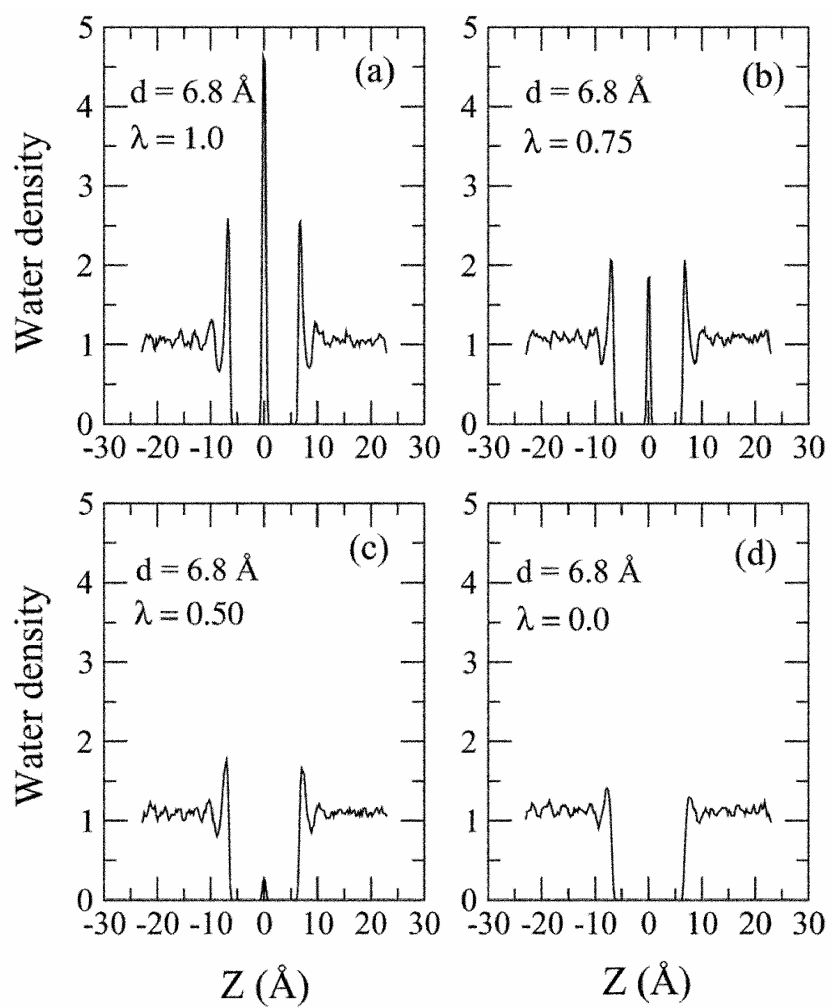

Figure 2. The density profiles of water in the vicinity of two single-layer graphite plates with an interplate separation of $6 \cdot 8 \AA$ and for different values of $\lambda$ as shown in the figures.

the simulation for $100 \mathrm{ps}$ for equilibration during which the temperature was kept fixed at $298 \mathrm{~K}$ through re- scaling of velocities. Upon equilibration, each system was further run for a production phase of 100-200 ps depending on system size with occasional rescaling of velocities to maintain the average temperature of $298 \mathrm{~K}$ and we used this production phase of the simulation for calculation of the density profiles and hydrogen bond properties of water molecules in the intervening region between the two plates and also in the regions near the outer surfaces of the plates.

\section{Results and discussion}

In figure la, we have shown the carbon-water interaction potentials for three different values of the tuning parameter of the solute-water attractive potential: $\lambda=1,0.5$ and 0 . In figure $1 \mathrm{~b}$, we have shown the corresponding carbon-oxygen radial distribution functions as obtained from simulations of a single carbon atom in water for these three different values of the tuning parameter. It is seen that in all cases, even when the carbon-water interaction is fully repulsive, there is enhanced density of water in the first hydration shell near the carbon atom as compared to that in the bulk phase. In particular, the density profiles are very similar for $\lambda=1$ and $0 \cdot 5$. Thus, the attractive dispersion interaction plays only a minor role in determining the solvation structure of small hydrophobic solutes, their hydration structures are primarily determined by the repulsive part of the potential. On the contrary, when the solvation structures of graphite plates made of many carbon atoms are concerned, dramatic differences are found 
for different values of the tuning parameter as can be seen from figure 2. Here we have shown the density profiles of water in the region between the two single-layer graphite plates with an interplate separation of $6 \cdot 8 \AA$ and also in the regions outside the two plates. The density profiles are computed from the simulation trajectory by taking a rectangular slab around the centre of the simulation box with its $x$ - and $y$-dimensions taken as the same as those of the graphite plates and its $z$-dimension taken to be the same as the length of the simulation box along the $z$ di-
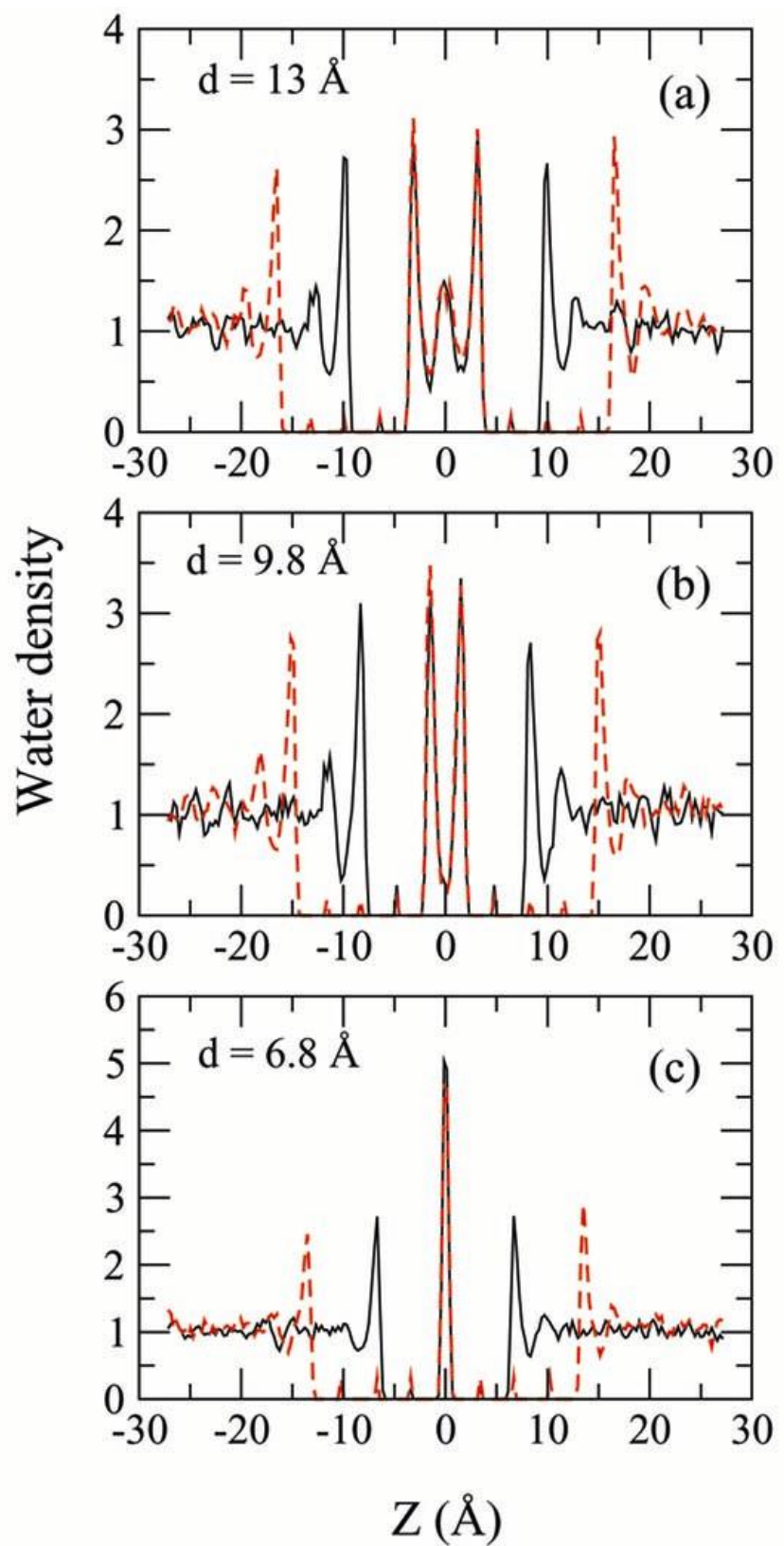

Figure 3. The density profiles of water in the vicinity of two graphite plates with an interplate separation of (a) $13 \AA$, (b) $9.8 \AA$ and (c) $6.8 \AA$. The solid (black) curves are for singlelayer and the dashed (red) curves are for triple-layer graphite plates. The value of $\lambda$ is 1 for all the systems. rection. It is seen that, depending on the value of $\lambda$, very different density profiles are found both in the intervening region between the two plates and also outside the plates. Especially, a complete dewetting is observed for the inner region for the fully repulsive potential and wetting is observed only when $\lambda=1$ or 0.75 . Thus, when the strength of the atom-water attractive dispersion interaction is not too small, the collective effects of the large number of such dispersion interactions stabilize the water layers between the two nanoscopic plates or near the outer surfaces and wetting of the plates is observed. These results are in agreement with the observations of Chowdhury and Pettitt (2005a, b), although the tuning of the attractive

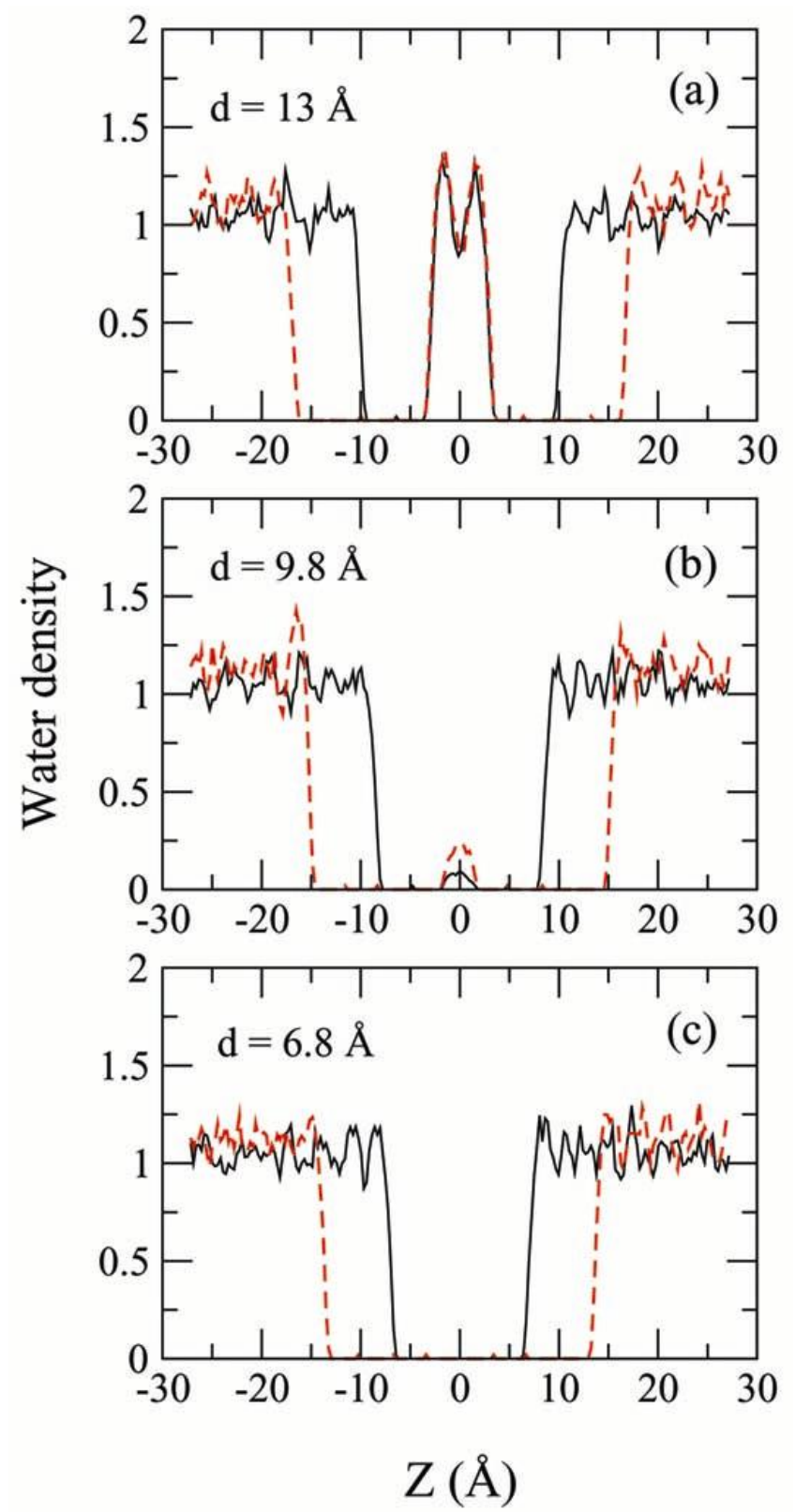

Figure 4. The density profiles of water in the vicinity of two graphite plates for $\lambda=0$. The different curves are as shown in figure 3 . 
strength of the carbon-water interaction potential was done differently in the latter work.

In figure 3, we have shown the effects of thickness and interplate separation on the solvation of graphite plates for $\lambda=1$. The corresponding results for $\lambda=0$ are shown in figure 4 . For the fully Lennard-Jones potential $(\lambda=1)$, the wetting of the plates are seen for all the three interplate separations considered here. Only a single layer of water molecules are found between the two plates for the smallest separation of $d=6.8 \AA$ whereas three layers of water could be accommodated in the confined region for $d=13 \AA$. Overall, the results for the single-layer plates are found to be very close to those for the three-layer

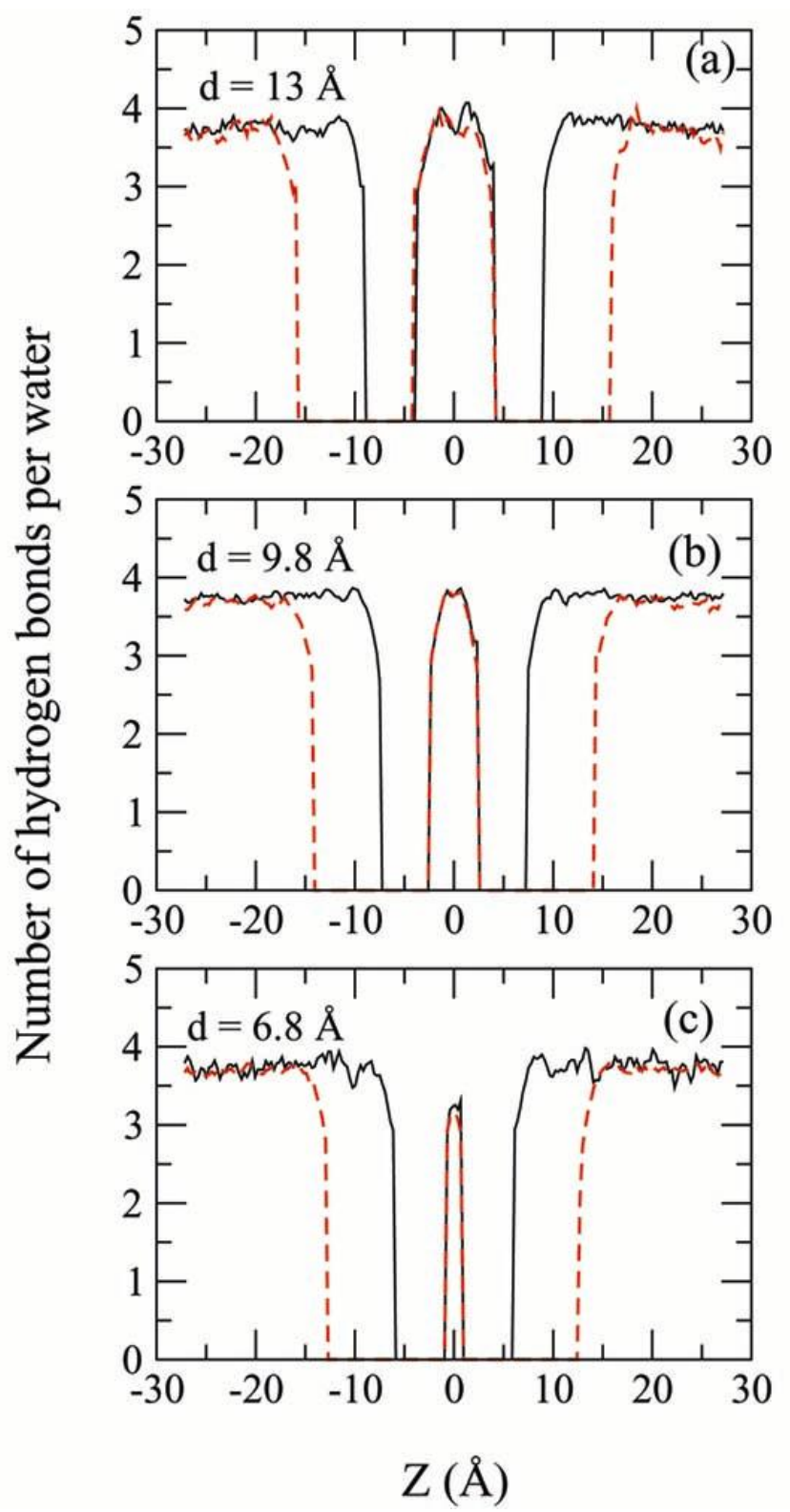

Figure 5. The number of hydrogen bonds per water molecule in the vicinity of two graphite plates for $\lambda=1$. The different curves are as in figure 3. plates. Thus, the thickness of the plates seems to play a minor role in determining the water structure near hydrophobic plates. For the fully repulsive carbon-water interactions $(\lambda=0)$, the water structure is very different between the two plates and also near the outer surfaces. Here, complete dewetting is observed for the smallest separation for both single and triple-layer plates and, only for largest separation of $13 \AA$, a significant filling of the confined region by water molecules is observed. Even then, the density profiles of water both inside and outside the graphite plates are found to be significantly different

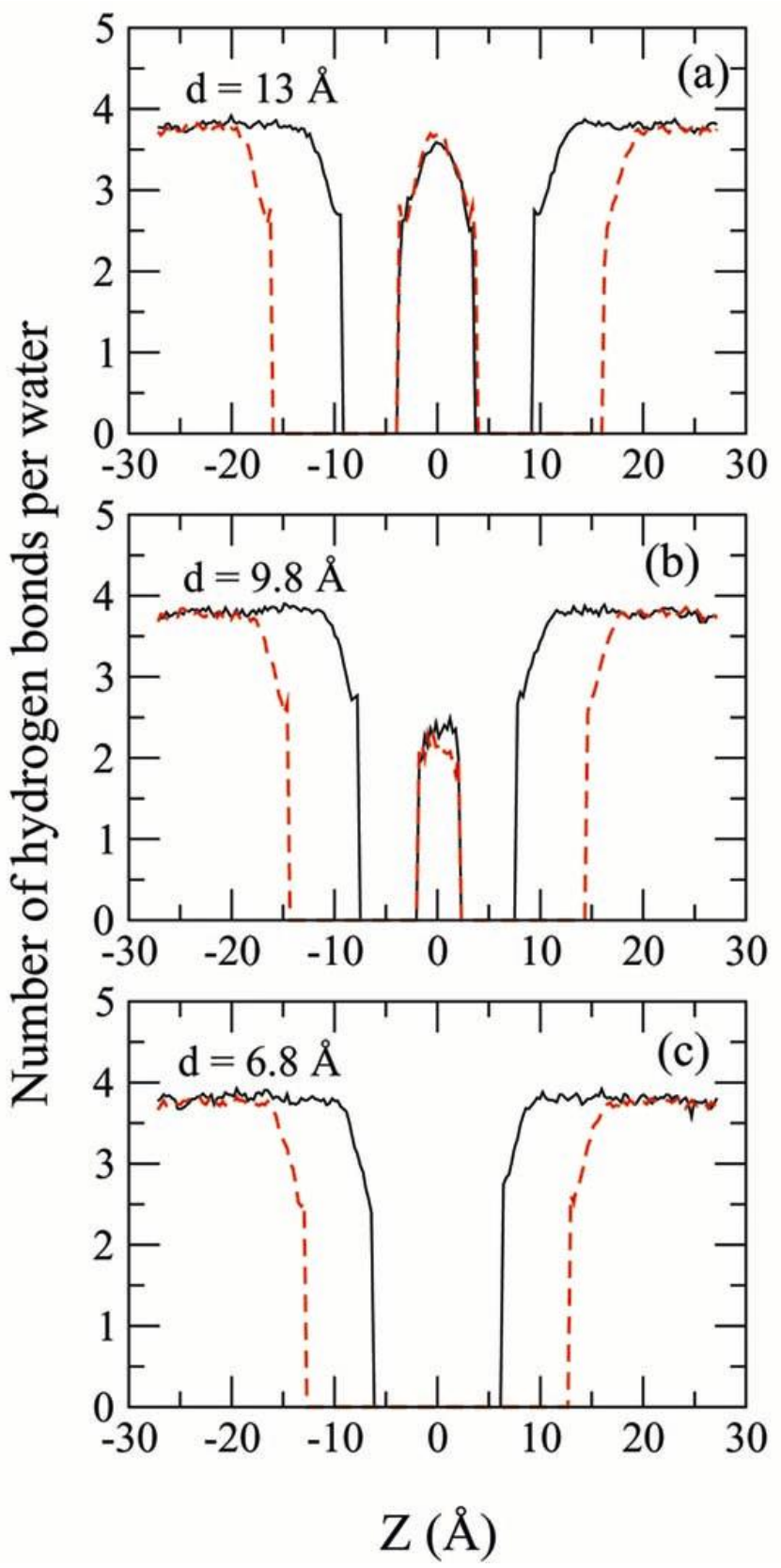

Figure 6. The number of hydrogen bonds per water molecule in the vicinity of two graphite plates for $\lambda=0$. The different curves are as shown in figure 3 . 

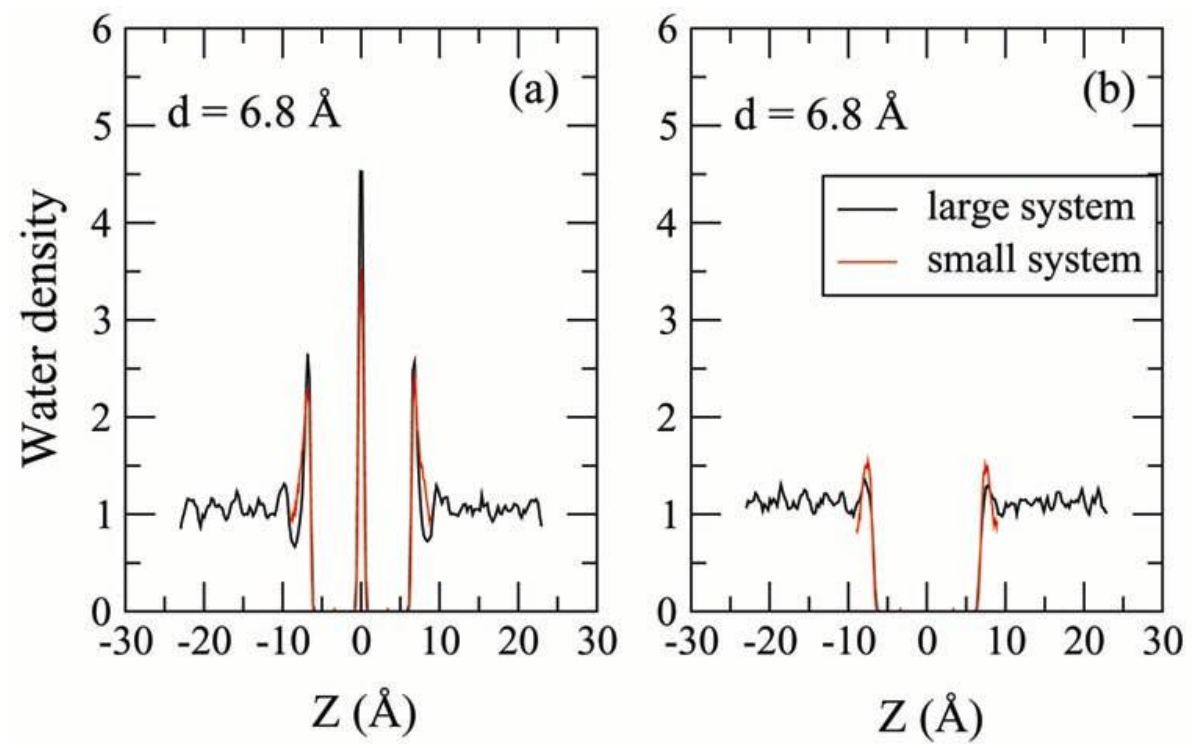

Figure 7. The density profiles of water in the vicinity of two single-layer graphite plates with an interplate separation of $6.8 \AA$ for two different systems sizes and for (a) $\lambda=1$ and (b) $\lambda=0$. Further details are as given in the text.

from those for $\lambda=1$ (figure 3 ). The hydrogen bond distributions of the systems of figures 3 and 4 are shown in figures 5 and 6 , respectively. We have used a geometric definition of the hydrogen bonds as was done in earlier studies (Luzar and Chandler 1996a, b; Chowdhury and Pettitt 2005a; Chowdhury and Chandra 2006; Mallik and Chandra 2006). We have, however, used only distance criteria and no angular cut-off was used. The number of hydrogen bonds per water molecule is found to be significantly reduced in confined region for the smallest interplate separation. Again, the effects of thickness is found to be minimal on the hydrogen bonded network on both sides of the plates.

Clearly, solvation structure of graphite-like hydrophobic plates are found to depend rather strongly on the nature of interaction potentials and the true interaction potential between a graphite plate and water are not known. Thus, in order to understand the true nature of hydrophobic interactions and water structure near hydrophobic surfaces, it is desirable to go beyond the use of empirical potentials and investigate these systems by means of $a b$ initio molecular dynamics simulations (Car and Parrinello 1985), where the many-body interaction potential and forces are computed through on-the-fly quantum electronic structure calculations. Since such calculations are computationally more expensive by several orders of magnitude, bigger systems of the type presented here in figures 1-6 could not be handled at present even by using good multiprocessor parallel computing facilities. Thus, it is important to know if the wetting/dewetting behaviour that is observed for the bigger systems will also hold for relatively smaller systems of, say, a few hundred atoms. In this regard, we have simulated a smaller system consist- ing of two single-layer graphite plates of 28 atoms each and 148 water molecules (after removing the overlapping molecules) and the results for the smaller systems are shown in figure 7 for both $\lambda=1$ and $\lambda=0$. For comparison, we have also included the results of figures $2 a$ and $d$ which were obtained for a larger system. It is seen that the main characteristics of hydrophobic solvation, i.e. wetting for $\lambda=1$ and dewetting for $\lambda=0$, are also reproduced by the smaller system.

\section{Conclusions}

In this work, we have investigated the structure of water in the vicinity of two hydrophobic plates of different thicknesses, interplate separation and interaction potentials. Results are also presented for different sizes of the simulation systems. In particular, we have considered two graphite-like plates immersed in liquid water with tunable strength of the attractive part of the carbon-water dispersion interaction. It is found that although the tuning of the attractive part of the interactions plays a minor role in the structure of water near a small carbon atomic solute, it plays a significant role in determining the structure of water near graphite-like plates made of many such atoms. Depending on the interplate separation and strength of the attractive interactions, different behaviours from wetting to dewetting of the region confined between the two plates are observed. Our calculations with single-layer versus triple-layer plates show that a variation in the thickness of the plates does not alter the density profiles and hydrogen bond distributions in any significant manner. This is true for the structure of intervening water between the two plates and also near the outer surfaces of the plates. 
Although majority of our calculations are done with rather large systems consisting of a few thousand atoms, some additional calculations with different sized systems reveal that smaller systems with a total of about five hundred atoms can also exhibit the essentials of wetting and dewetting behaviour on changes of the attractive strength of the carbon-water interaction potential. This is significant because it may be possible to handle such smaller systems by means of $a b$ initio molecular dynamics methods without involving any predefined empirical potentials and such calculations are expected to provide a better understanding of the molecular nature of hydrophobic solvation in aqueous systems containing nanoscopic graphite plates.

\section{Acknowledgements}

We thank the Department of Science and Technology (DST), Government of India, for financial support and the University Grants Commission (UGC) for a research fellowship to (MR). Part of the calculations was done in the Computer Centre, Indian Institute of Technology, Kanpur.

\section{References}

Allen M P and Tildesley D J 1987 Computer simulation of liquids (Oxford: Clarendon Press)
Beckstein O and Sansom M S P 2003 Proc. Natl. Acad. Sci. 100 7063

Berendsen H J C, Grigera J R and Straatsma T P 1987 J. Phys. Chem. 916269

Car R and Parrinello M 1985 Phys. Rev. Lett. 552471

Choudhury N and Pettitt B M 2005a J. Am. Chem. Soc. 127 $3556 ; 1294847$

Choudhury N and Pettitt B M 2005b Mol. Simul. 31457

Chowdhuri S and Chandra A 2006 J. Phys. Chem. B110 9674

Cornell D W et al 1995 J. Am. Chem. Soc. 1175179

Gordillo M C and Marti J 2002 J. Chem. Phys. 1173425

Huang D M and Chandler D 2000 Proc. Natl. Acad. Sci. 97 8324

Huang D M and Chandler D 2002 J. Phys. Chem. B106 2047

Huang X, Margulis C J and Berne B J 2003 Proc. Natl. Acad. Sci. 10011953

Hummer G, Garde S, Garcia A E and Pratt L R 2000 Chem. Phys. 258349

Hummer G, Rasaiah J C and Noworyta J P 2001 Nature 414 188

Lum K, Chandler D and Weeks J D 1999 J. Phys. Chem. B103 4570

Luzar A and Chandler D 1996a Phys. Rev. Lett. 76928

Luzar A and Chandler D 1996b Nature (London) 37955

Mallik B S and Chandra A 2006 J. Chem. Phys. 125234502

Smith D E and Haymet A D J 1993 J. Chem. Phys. 986445

Wallqvist A, Gallicchio E and Levy R M 2001 J. Phys. Chem. B105 6745 